Journal for ImmunoTherapy of Cancer

\section{Pickering emulsions with ethiodized oil and nanoparticles for slow release of intratumoral anti-CTLA4 immune checkpoint antibodies}

\author{
Lambros Tselikas (1) , ${ }^{1,2}$ Thierry de Baere, ${ }^{1}$ Thomas Isoardo, ${ }^{2}$ Sandrine Susini, ${ }^{2}$ \\ Karine Ser-Le Roux, ${ }^{3}$ Mélanie Polrot, ${ }^{3}$ Julien Adam, ${ }^{4}$ Mathieu Rouanne, ${ }^{2}$ \\ Laurence Zitvogel, ${ }^{5}$ Laurence Moine, ${ }^{6}$ Fréderic Deschamps, ${ }^{1}$ \\ Aurélien Marabelle (1D) 2,7
}

To cite: Tselikas L, de Baere T, Isoardo T, et al. Pickering emulsions with ethiodized oil and nanoparticles for slow release of intratumoral antiCTLA4 immune checkpoint antibodies. Journal for ImmunoTherapy of Cancer 2020;8:e000579. doi:10.1136/ jitc-2020-000579

- Additional material is published online only. To view please visit the journal online (http://dx.doi.org/10.1136/jitc2020-000579).

Accepted 17 April 2020

\section{Check for updates}

(c) Author(s) (or their employer(s)) 2020. Re-use permitted under CC BY-NC. No commercial re-use. See rights and permissions. Published by BMJ.

For numbered affiliations see end of article.

\section{Correspondence to} Dr Lambros Tselikas; lambros.tselikas@ gustaveroussy.fr

\section{ABSTRACT}

Background Intratumorous immunotherapy for cancer is currently thriving. The aim of such local strategy is to improve the therapeutic index of these treatments, for higher on-target/on-tumor activity and less on-target/offtumor adverse events. Strategies allowing for slow release of anti-CTLA4 in the tumor microenvironment could improve their clinical efficacy.

The purpose of the study was to develop a radiopaque delivery platform to improve the targeting and exposure of intratumorous anti-CTLA4 antibodies for cancer immunotherapy.

Methods Pickering emulsions of anti-CTLA4 antibodies were formulated with radiopaque ethiodized oil and poly-lactic-co-glycolic acid (PLGA) nanoparticles. We characterized the microscopic aspect and stability of such emulsions using Turbiscan. We monitored the release of anti-CTLA4 over time from these emulsions and evaluated their structure using mass spectrometry. We then tested the functionality of the released antibodies by preforming ex vivo competitive binding assays. Finally, we assessed the in vivo efficacy of intratumorous anti-CTLA4 Pickering emulsions.

Results Pickering emulsions of ethiodized oil and PLGA nanoparticles (PEEPs) resulted in a radiopaque waterin-oil emulsion with average internal phase droplet size of $42 \pm 5 \mu \mathrm{m}$ at day 7 . Confocal microscopy showed that anti-CTLA4 antibodies were effectively encapsulated by ethiodized oil with PLGA nanoparticles located at the interface between the aqueous and the oily phase. Turbiscan analysis showed that emulsions were stable with continuous and progressive release of anti-CTLA4 antibodies reaching $70 \%$ at 3 weeks. Structural and functional analysis of the released antibodies did not show significant differences with native anti-CTLA4 antibodies. Finally, intratumorous anti-CTLA4 PEEPs were able to eradicate tumors and cure mice in a syngeneic immunocompetent preclinical tumor model. Conclusion Pickering emulsions of ethiodized oil and PLGA is an innovative radiopaque delivery platform that does not alter the functionality of anti-CTLA4 immune checkpoint antibodies. Beyond local anti-CTLA4 applications, these emulsions might be used with other therapeutic molecules for optimal intratumorous or intraarterial delivery of novel cancer immunotherapies.

\section{INTRODUCTION}

Over the past decade, immunotherapies have dramatically changed the landscape of cancer treatment. Ipilimumab, an $\mathrm{IgG}_{1}$ antiCTLA4 antibody, has been the first immune checkpoint-targeted immunotherapy approved for the treatment of metastatic melanoma. It was shown to provide a significant improvement in the overall survival of this deadly disease, with even cure of metastatic disease, for about $20 \%$ of the patients. ${ }^{1-3}$

Anti-CTLA4 antibodies present with characteristics that justify further research on their delivery to improve their therapeutic index. As opposed to anti-PD(L) 1 antibodies where no correlation between dose, efficacy, and toxicity has been observed, ${ }^{45}$ anti-CTLA4 antibodies have a significant dose-effect correlation on both efficacy ${ }^{6}$ and toxicity. ${ }^{7}$ Interestingly, in pharmacokinetic studies, the efficacy of systemic anti-CTLA4 therapy has also been shown to be dependent from the exposure to the treatment. ${ }^{8}$ Also systemic exposure to anti-CTLA4 antibodies can generate up to $28 \%$ of CTCAE grade 3-5 immune-related adverse events (irAEs) in mature clinical trials at a dose of $3 \mathrm{mg} / \mathrm{kg}$ every 3 weeks ${ }^{9}$. Moreover grade 3-5 irAEs are increased to $59 \%$ when anti-CTLA4 is combined to anti-PD-1. ${ }^{10}$ Therefore, there is a call for alternative treatment modalities which could increase the anti-CTLA4 bioavailability inside the tumor while keeping systemic exposure as low as possible to improve efficacy and decrease toxicity.

A promising treatment strategy is 'in situ immunization' which consists in 
intratumorous injection of immunostimulatory products such as immunostimulatory monoclonal antibodies (ISmAbs) or viruses. ${ }^{11}$ ISmAbs or viruses are injected directly into a tumor to locally prime the antitumor immune response. ${ }^{12}$ Interestingly, low doses of monoclonal antibodies (ISmAbs) delivered directly into the tumor can be sufficient to generate a systemic antitumor immune response able to eradicate distant, not injected (anenestic), tumor sites. ${ }^{13} 14$ Hence, local delivery of immunotherapies is an appealing strategy to decrease the autoimmune and inflammatory toxicities observed on systemic delivery while improving the therapeutic index of such treatments. Accordingly, many 'in situ immunization' clinical trials are currently ongoing. ${ }^{15}$ Moreover, preclinical and clinical data have also recently provided the rationale to local anti-CTLA4 therapy, showing both local and abscopal efficacy results. ${ }^{13} 16-20$

Nevertheless, percutaneous intratumorous local injections of ISmAbs have some limitations. First, tumors might need to be punctured repetitively, for example every week or 2 weeks, because there is no sustained local release of the injected ISmAbs, and tumors that can be punctured safely in such a repetitive manner are mostly superficial. Second, the spatial distribution of ISmAbs inside of the targeted tumor may be inhomogeneous and inappropriate, with antibodies concentrating mostly within the low-pressure necrosis areas of the tumor. Third, there is no monitoring, during or after injection, of the distribution of the ISmAbs within the tumor or outside the tumor with potential leaks. To overcome some of these limitations, the use of radiopaque delivery platforms may be of interest as they allow for sustained release, delivery monitoring with X-ray-based imaging, and ideally can be administrated via various routes to the tumor (percutaneous, intra-arterial, intralymphatic).

Theoretically, the formulation process of an emulsion offers the possibility to encapsulate a large spectrum of hydrophilic drugs within an oily phase. Standard emulsions using radiopaque ethiodized oil have been used for years to deliver chemotherapeutic agents to liver tumors. ${ }^{21}$ For instance, hepatic arterial delivery is the most common administration route to take advantage of the arterial phase hyperenhancement of hepatic tumors. $^{22-24}$

Recently, we successfully developed a biocompatible Pickering emulsion, using ethiodized oil and biodegradable nanoparticles (NPs) made of poly-lactic-co-glycolic acid (PLGA), and demonstrated in preclinical models a slower release of oxaliplatin ${ }^{25}$ and doxorubicin ${ }^{26} 27$ compared with standard emulsions.

The aim of this study was to evaluate such ethiodized oil and PLGA Pickering emulsions as a radiopaque drug delivery platform to improve the targeting and treatment exposure profile of anti-CTLA4 antibodies for cancer local immunotherapy.

\section{MATERIALS AND METHODS}

First, we formulated and compared standard and Pickering emulsions of anti-CTLA4. Then, we evaluated the physical characteristics of anti-CTLA4 antibodies released from the emulsion to assess any changes compared with 'native' antibodies. Finally, we assessed the functionality of the released anti-CTLA4 antibodies in an ex vivo competitive binding assay on a cell line and in vivo using transplantable colon cancer bearing mice.

\section{Emulsions of anti-CTLA4}

Based on previous publications, ${ }^{26}{ }^{27}$ we aimed to formulate a radiopaque water-in-oil emulsion able to deliver the anti-CTLA4 antibody into the tumor in a progressive manner. For this purpose, we formulated anti-CTLA4 standard emulsions with ethiodized oil and Pickering emulsions using ethiodized oil and PLGA NPs (PEEP).

\section{Emulsion formulation}

For standard emulsion and PEEP, the oily phase was composed of ethiodized oil (Lipiodol, Guerbet, France) and the aqueous phase was composed of an anti-CTLA4 antibody (ipilimumab; Yervoy, BMS, USA), at a concentration of $5 \mathrm{mg} / \mathrm{mL}$. The Pickering emulsion contained biocompatible and biodegradable PLGA NPs ${ }^{27}$ (online supplementary data figure 1 ).

\section{Location of anti-CTLA4 within the emulsions}

Emulsions were analyzed by optical microscopy (BX60F, Olympus, Japan) at $100 \times$ magnification. To evaluate the location of anti-CTLA4 antibodies within the emulsions, we used confocal microscopy (META LSM 510 confocal microscope; Zeiss, Germany). Ipilimumab was conjugated prior with Alexa Fluor 647 (AF647), then labeled antibody was further diluted 100 times with non-conjugated ipilimumab (1\% AF647-conjugated ipilimumab). To label the NPs, we first linked covalently PLGA with rhodamine. PLGA-rhodamine was added during the formulation of PLGA NPs to form PLGA NPs $(0.1 \%$ rhodamine $)$.

\section{Emulsion stability}

To evaluate the stability of the emulsions, we measured the size of the internal phase aqueous droplets over time and we measured the time for complete coalescence.

Droplets size measurement was determined at day 1 , 7, 14, and 35 after formulation, using a particle counter, shape, and size analysis apparatus (Flowcell FC200S+HR; Occhio, Belgium). Emulsions passed through a $400 \mu \mathrm{m}$ spacer and diluted 20 times in ethiodized oil before analysis. Each emulsion was measured five times with at least 1000 droplets analyzed by measurement.

Time for complete coalescence of both emulsions was obtained using a Turbiscan (Classic MA 2000 apparatus; Formulation, Toulouse, France). A near-infrared light to scan the emulsions and calculated the transmitted and scattered light. Measurements were repeated every hour for the first 24 hours, and at day 7 and day 14 after formulation at $25^{\circ} \mathrm{C}$. Emulsion stability was defined as the absence of difference for the backscattering diffusion 
between two measurements, excepted for the creaming phenomenon that is reversible by hand shaking.

Emulsions were stored in glass vials, away from light at a temperature at $25^{\circ} \mathrm{C}$.

\section{Ex vivo evaluation of released anti-CTLA4 antibodies Anti-CTLA4 antibody release}

The release of anti-CTLA4 antibodies was quantified using micro-bicinchoninic acid assay technique at days 1 , $3,6,10,15,21$, and 28 after formulation. A total of $0.8 \mathrm{~mL}$ of emulsion (corresponding to $1 \mathrm{mg}$ of ipilimumab) was deposited into $20 \mathrm{~mL}$ of phosphate-buffered saline (PBS) as release medium, and $1 \mathrm{~mL}$ of release medium was then withdrawn at each time point and replaced by fresh release medium.

To assess whether antibodies released from the emulsion were intact, we compared their physical characteristics with those of the same native ipilimumab antibody, not used for the emulsions.

Sizes of antibodies were compared by means of electrophoresis gel migration. We used Mini-PROTEAN TGX stain-free precast gels (BioRad, USA) and the size was determined with the PageRuler Plus prestained protein ladder (Thermo Fisher Scientific, USA). A total of $15 \mu \mathrm{L}$ of the release samples collected at the different time points were compared with the same volume of native ipilimumab antibodies.

Capillary zone electrophoresis-mass spectrometry (CZE-MS) experiments were performed using a 7100 Agilent capillary electrophoresis, ESI-QTOF 6540 mass spectrometer (Agilent Technologies, Santa Clara, California, USA) and Mass Hunter B.07.00 software. Analysis of ipilimumab were performed on the triple-layer coated capillary of Polybrene-dextran sulfate-Polybrene. ${ }^{28}$

\section{Released anti-CTLA4 antibody functional evaluation}

To assess the functionality of the released anti-CTLA4 antibodies, we performed an ex vivo competitive binding assay on a cell line, and then an in vivo mouse experiment.

\section{Ex vivo competitive binding assay}

The CTLA4 Ig-24 (ATCC CRL-10762) (ATCC, USA) cell line has been generated from ovarian hamster cells transfected to express in their cytoplasm the soluble CTLA4-Ig fusion protein, which contains the extracellular domain of the CTLA4 human protein. These cells were cultured at $37^{\circ} \mathrm{C}$ in $5 \% \mathrm{CO}_{2}$ in Dulbecco's modified Eagle medium completed with $10 \%$ of fetal bovine serum, $1 \%$ of glutamine, $1 \%$ of penicillin and streptomycin, $0.2 \mathrm{mM}$ of proline and $1 \mu \mathrm{M}$ of methotrexate. Before the experience, we tagged native ipilimumab with AF647 fluorophore (Alexa Fluor 647 Antibody Labeling Kit; Thermo Fisher Scientific, USA) for detection with flow cytometry (FACS). First, the cells were permeabilized and fixed thanks to BD Cytofix/Cytoperm solution. The cells were then treated either with native ipilimumab or with the PEEP-released ipilimumab during $30 \mathrm{~min}$ at $4^{\circ} \mathrm{C}$. Then, the AF647-conjugated ipilimumab was added in all conditions. After this second incubation, fluorescence was measured using flow cytometry (LSR Fortessa X20, BD Biosciences, USA).

In vivo anti-CTLA4 functional assessment

The antitumorous activity of the anti-CTLA4 antibodies was assessed in vivo, on 6-week-old female BALB/c mice purchased from Charles River Laboratories (France). For this experiment, we used an anti-mouse $\operatorname{IgG}_{2 \mathrm{~b}}$ antiCTLA4 (Clone: 9H10, Bio X Cell, USA) as well as its isotype.

CT26 ATCC CRL-2638 (ATCC, USA) cells, a tumor cell line of colorectal cancer responding to anti-CTLA4 therapy, ${ }^{29}$ were cultured in Dulbecco's modified Eagle medium with GlutaMAX completed with $10 \%$ of fetal bovine serum, $1 \%$ of penicillin and streptomycin, and $1 \%$ of non-essential amino acids at $37^{\circ} \mathrm{C}$ in $5 \% \mathrm{CO}_{2}$. Just before injection, the tumor cells were collected and suspended at a concentration of $1.10^{6}$ cells $/ 100 \mu \mathrm{L}$ in PBS at $4^{\circ} \mathrm{C}$. The tumor cells $\left(\cong 1.10^{6}\right)$ were injected subcutaneously under isoflurane $2 \%$ sedation.
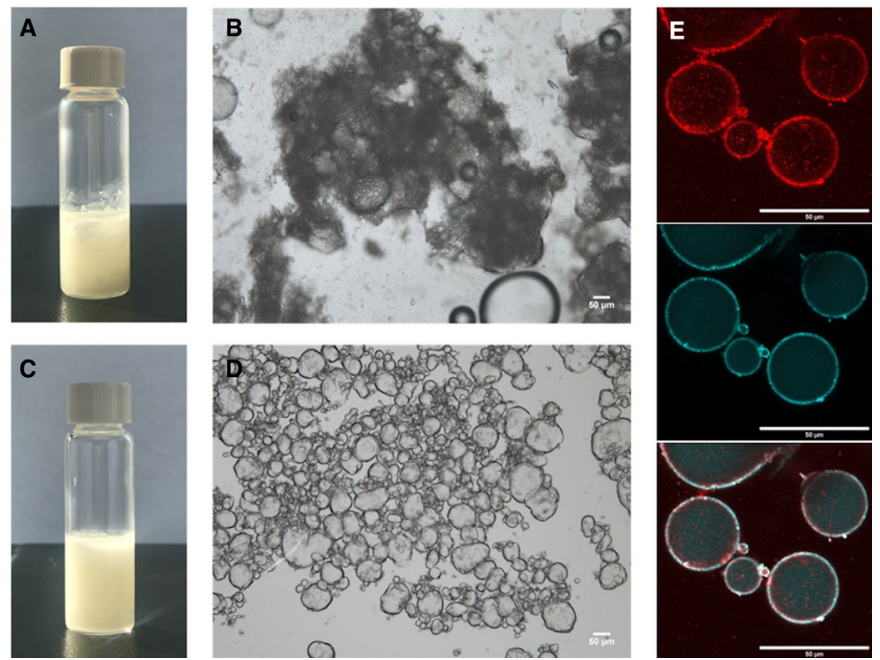

Figure 1 . Macroscopic and microscopic analyses of ethiodized oil and poly-lactic-co-glycolic acid (PLGA) Pickering emulsions. (A) Immediate coalescence (flocculation) after formulation of standard anti-CTLA4 emulsion without PLGA nanoparticles (NPs). (B) Corresponding microscopic appearance of the emulsion showing the antibody aggregation and phase separation just after formulation (scale bar: $50 \mu \mathrm{m})(\mathrm{C})$ Homogeneous anti-CTLA4 Pickering emulsion using ethiodized oil together with PLGA NPs (D.) Microscopic analysis of corresponding emulsion showing the presence of aqueous phase droplets. Pickering emulsion on day 1 after formulation (scale bar: $50 \mu \mathrm{m}$ ) showing a 'water-inoil' aspect. (E) Confocal microscopy of Pickering emulsion on day 1 after formulation using AF647-conjugated ipilimumab (light blue) and rhodamine-conjugated PLGA NPs (red) showing that nanoparticles located at the interface between the oily phase (ethiodized oil) outside and the aqueous phase (ipilimumab) inside (scale bar: $50 \mu \mathrm{m}$ ). The experiment has been conducted several times yielding similar results. A typical and representative photograph is shown. 
Treatment was performed at day 6 after inoculation, in 32 mice when tumors where palpable and measure more than $50 \mathrm{~mm}^{3}$. In 10 mice, a constant volume of $50 \mu \mathrm{L}$ of Pickering emulsion was injected directly into the tumor at a dosage of $100 \mu \mathrm{g}$ of anti-CTLA4. As a positive control, 10 mice received intratumorous injection of $100 \mu \mathrm{g}$ of native (unemulsified) anti-CTLA4 diluted in $50 \mu \mathrm{L}$ of PBS. Negative control groups of 12 mice were injected either with a Pickering emulsion containing the anti-CTLA4 isotype $(n=5)$ or the isotype only $(n=7)$. In case of durable or complete response lasting at 6 weeks, a rechallenge was performed on the opposite flank with $1.10^{6}$ CT26 tumor cells to assess if a systemic specific antitumorous immunization was achieved. For the rechallenge experiment, five additional mice were inoculated as a positive control to confirm the capacity of the cell line to develop tumors.

For all in vivo experiments, mice were monitored three times a week, and sacrificed either when the tumor reached $1500 \mathrm{~mm}^{3}$ or 3 months after initial injection. Usual additional endpoints such as tumor ulceration, weight loss $>10 \%$ or behavioral alteration were also used to guaranty animal well-being. X-ray three-dimensional imaging was performed before sacrifice using an IVIS Spectrum CT (Perkin Elmer, USA). Histopathological analysis was performed by a pathologist on formalin-fixed paraffin-embedded tumor tissue, using H\&E staining after sacrifice.

\section{Statistics}

Categorical variables were expressed as count and frequency. Qualitative analyses of ex vivo experiments were only descriptive. Continuous variables were expressed using medians and IQR and comparisons were done using the non-parametric Kruskal-Wallis test. In the in vivo experiment, survival rates were estimated using the Kaplan-Meier method, and HRs and 95\% CI were determined by Cox proportional hazards regression. All tests were two sided. A p-value lower than 0.05 was considered statistically significant. Analysis and graphs were done using Prism v8 (La Jolla, California, USA).

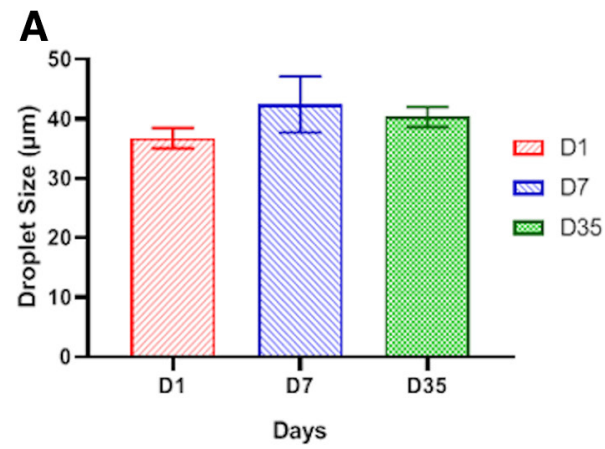

\section{RESULTS}

\section{Pickering emulsion formulation}

All standard anti-CTLA4 emulsions with ethiodized oil demonstrated immediate coalescence with a flocculation process, without stable droplet formation on microscopic analysis. Conversely, all PEEP (ethiodized oil+PLGANPs+anti-CTLA4 antibody) resulted in waterin-oil emulsions (figure 1).

Confocal microscopy showed the therapeutic agent being contained inside the droplets (AF647-conjugated ipilimumab), the NPs (labeled with rhodamine) being located at the interface between the aqueous and the oily phases and acting as a stabilizer (figure 1).

Turbiscan analysis confirmed that all Pickering emulsions remained stable at last time point, day 7 . The mean size of droplets was $37 \pm 3 \mu \mathrm{m}$ on day 1 . The size of droplets showed no significant variation over time $(42 \pm 5 \mu \mathrm{m}$ at day 7 and $40 \pm 5 \mu \mathrm{m}$ at day 35 ). No coalescence process was observed macroscopically and microscopically immediately after emulsion formulation, and when morphological analysis was repeated 1 week and 1 month later (figure 2).

\section{Ex vivo evaluation of released anti-CTLA4 antibodies}

Because of immediate coalescence, the release of ipilimumab from standard ethiodized oil emulsion was not evaluated. The release profile of ipilimumab from PEEP is shown on figure 3. The percentage of ipilimumab released from Pickering emulsions were 5.3 \pm 0.93 , $14.0 \pm 4.8,25.6 \pm 5.6,38.1 \pm 5.7,51.3 \pm 7.0,64.1 \pm 16.7$, and $66.6 \pm 11.5$ at day $1,3,6,10,15,21$, and 28 , respectively.

The protein migration profile of the released antibodies was similar to that of the native ipilimumab, with a main protein band at $150 \mathrm{KDa}$ (online supplementary data figure 2).

The CZE-MS analysis of native ipilimumab at a concentration of $5 \mathrm{mg} / \mathrm{mL}$ showed a peak at $8.4 \mathrm{~min}$ at which MS spectrum displayed the envelopes with a maximum mass-to-charge $(\mathrm{m} / \mathrm{z})$ at $4303.24 \mathrm{~m} / \mathrm{z}$ (figure $4 \mathrm{~B}$ ). For concentrated aliquot of release samples, the analysis also displayed a peak at $8.4 \mathrm{~min}$. In addition, the MS spectrum

Figure 2 . Stability of Pickering emulsions using ethiodized oil and poly-lactic-co-glycolic acid (PLGA) nanoparticles. (A) Average droplet size measurements \pm SEM (D50 by volume) of Pickering emulsions on days 1, 7, and 35. (B) Backscattering measurement of Pickering emulsions during 14 days. The creaming process, a reversible phenomenon, occurred during 7 days; otherwise no destabilization process such as coalescence was observed during the following days. The experiment has been conducted three times yielding similar results. A typical and representative curve is shown. 
A

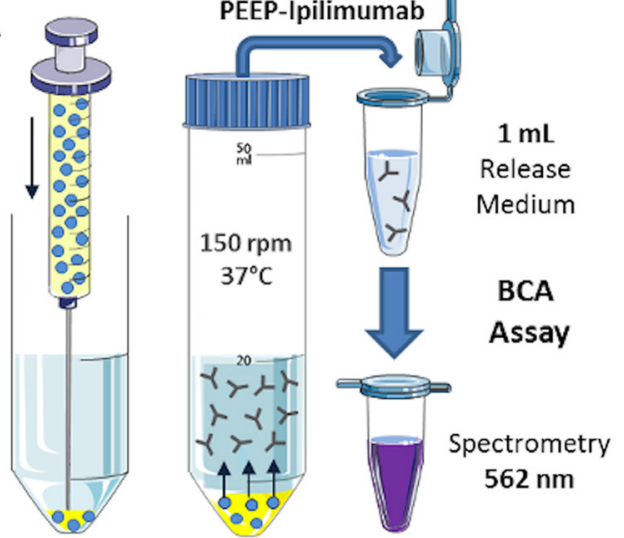

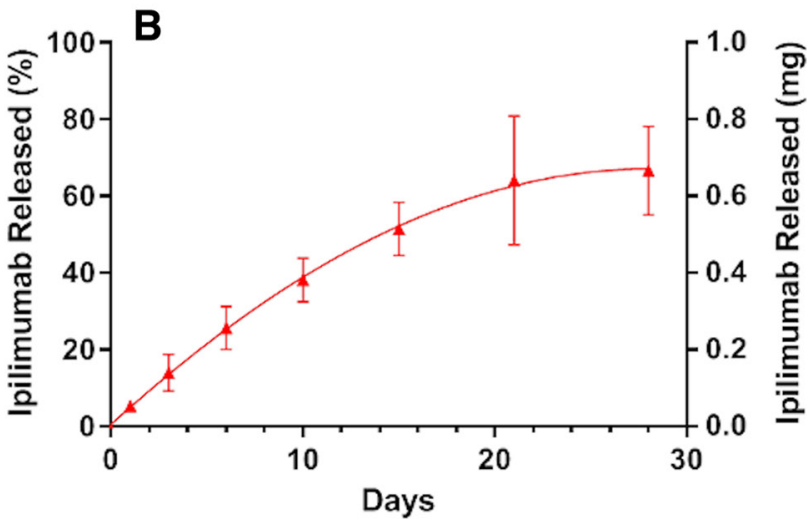

Figure 3 . Time-dependent release of ipilimumab from Pickering emulsions using ethiodized oil and poly-lactic-co-glycolic acid (PLGA) nanoparticles (PEEP). (A) Titration of ipilimumab release over time from ethiodized oil and PLGA Pickering emulsions (oil/water ratio: 3:1) using bicinchoninic acid (BCA) assay. (B) Percentage (left y-axis) and mass (right y-axis) of ipilimumab released over time by ethiodized oil and PLGA ipilimumab Pickering emulsions.

at this peak exhibited a charge state distribution centering at $35+(4197.93 \mathrm{~m} / \mathrm{z})$ that matches to the mass spectrum of native ipilimumab (figure 4C,D).

\section{Released anti-CTLA4 antibody functional evaluation}

The ex vivo competitive binding assay confirmed that both native anti-CTLA4 (ipilimumab) and the released antibodies (from PEEP) were able to bind to CTLA4 as shown in figure 5. Furthermore, the fluorescence shift was similar for native and PEEP-released anti-CTLA4.

We next tested these two local therapies in transplantable colon cancer syngeneic of BALB/c mice (CT26) after subcutaneous inoculation. In vivo anti-CTLA4 functionality assessment demonstrates a beneficial effect of local anti-CTLA4 antibodies with both modalities, with no significant difference in terms of survival between injection of PEEP and direct injection of anti-CTLA4. At the end of the experiment (after 6 weeks of follow-up), 8/10
$(80 \%)$ and $7 / 9(77.7 \%)$ mice were alive in the PEEP and direct injection arms respectively, $\mathrm{p}=0.95$. Median overall survival was not reached for both groups (HR=1.71; $95 \%$ CI 0.29 to $9.99, \mathrm{p}=0.54)$. One mouse died at day 2 after treatment in the direct injection group. Three and two mice had to be sacrificed before the end of the experiment in the group direct injection and PEEP group, respectively $(p=0.6)$. However, the median tumor volume was significantly higher in the PEEP group $\left(125 \mathrm{~mm}^{3} \mathrm{vs}\right.$ $\left.0 \mathrm{~mm}^{3}(\mathrm{p}<0.001)\right)$. Among responders in the direct injection group, all mice showed complete response. In the Pickering emulsion group, there was an initial tumor volume increase and then a decrease until a long lasting 'plateau', with no tumor regrowth during the 6 weeks of follow-up. Figure 6 summarizes the results in terms of tumor growths and survival (online supplementary data figure 3).
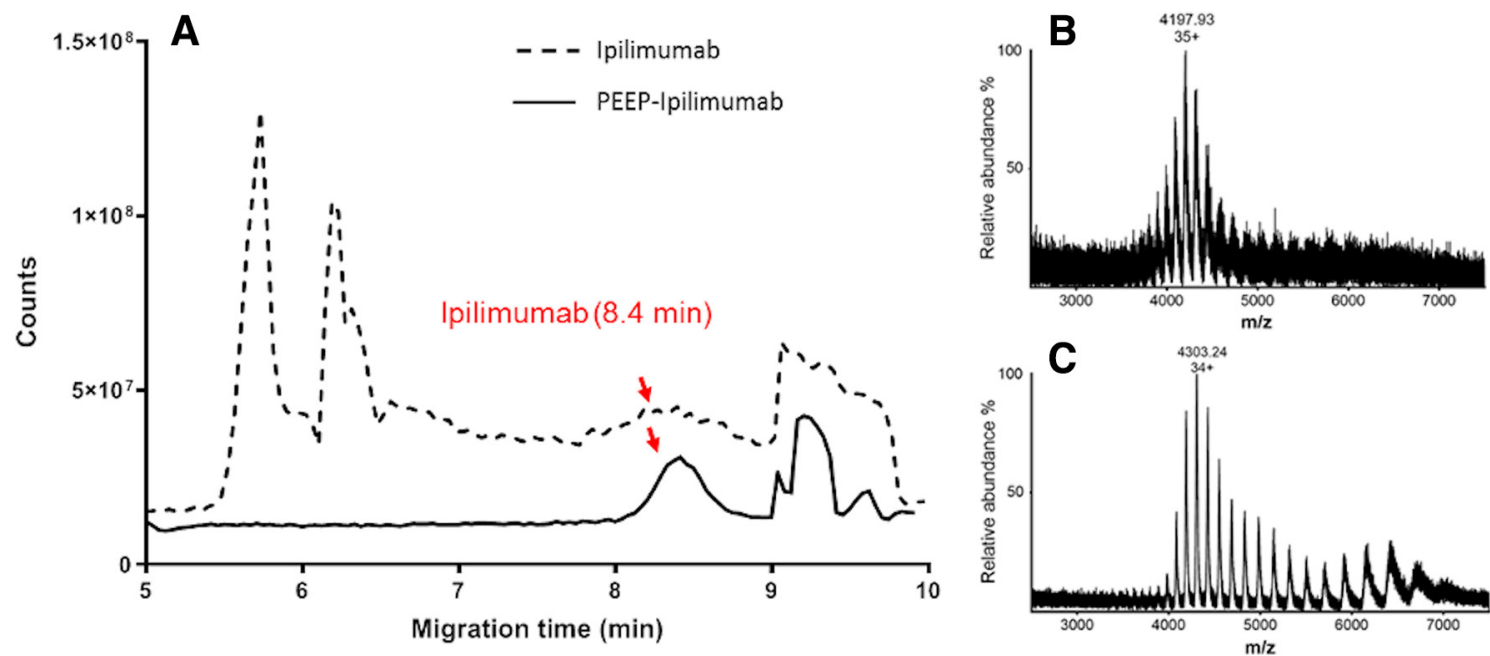

Figure 4 . Mass spectrum comparison between ipilimumab and ipilimumab released from Pickering emulsions using ethiodized oil and poly-lactic-co-glycolic acid nanoparticles. (A) Mass spectrum comparison of ipilimumab and PEEPsipilimumab obtained by capillary zone electrophoresis-mass spectrometry. Both commercial and PEEPs-released ipilimumab share the same migration time. (B and C) Focus on mass-to-charge $(\mathrm{m} / \mathrm{z}$ ) spectrum from ipilimumab and PEEPs-released ipilimumab. Both share a close $\mathrm{m} / \mathrm{z}$ value for the main peak and the same charge state distribution. 


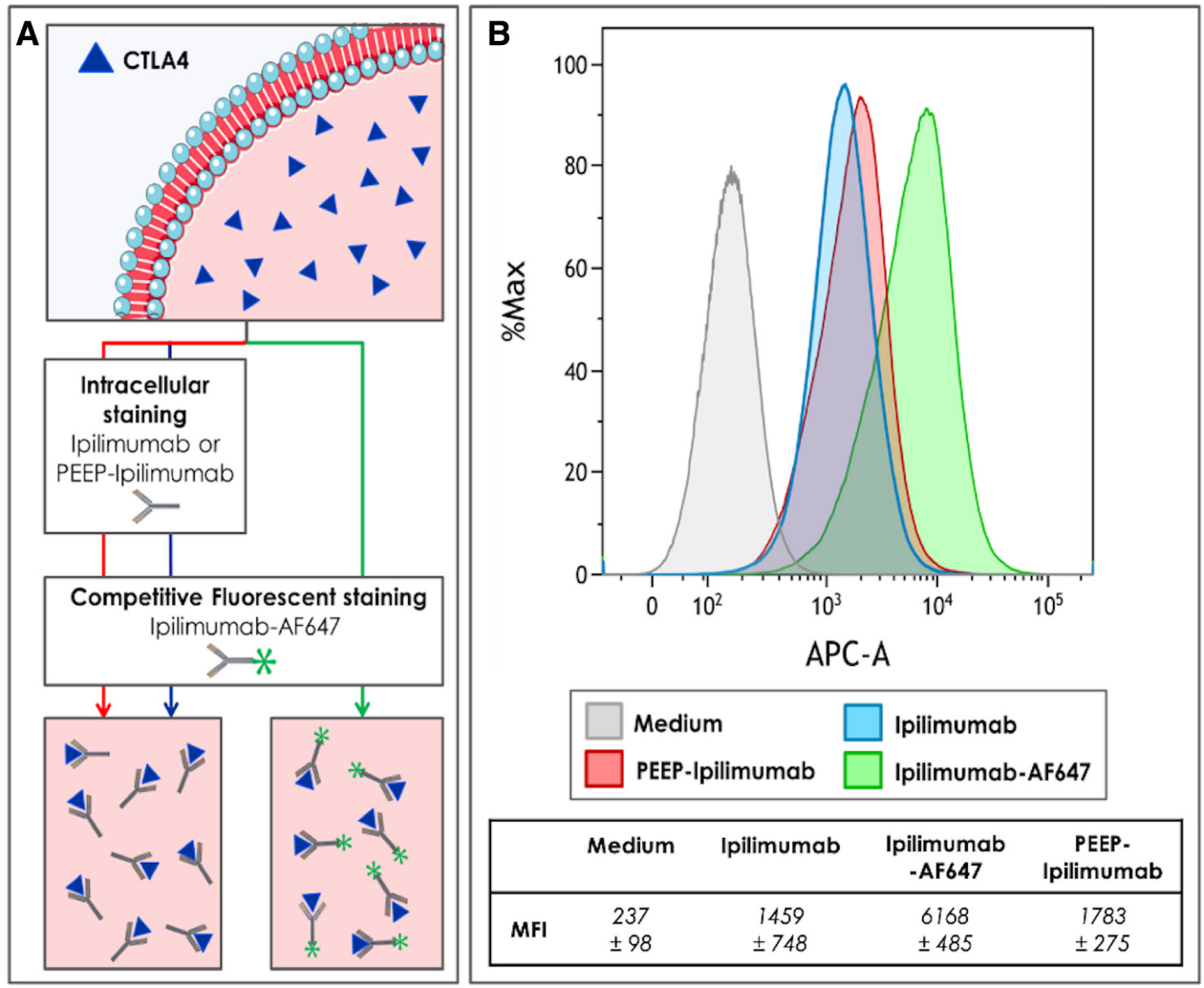

Figure 5 . Functional assessment of CTLA4 binding by ipilimumab released from Pickering emulsions using ethiodized oil and poly-lactic-co-glycolic acid nanoparticles (PEEP). (A) Design of competitive binding assay of ipilimumab and PEEP-ipilimumab on CTLA4-Ig transgenic cell line. PEEP-ipilimumab was collected after 21 days of release by the PEEP. Medium: non-treated cell; ipilimumab: addition of native ipilimumab and then Alexa Fluor 647 (AF647)-conjugated ipilimumab; PEEP-ipilimumab 21D: addition of release sample of ipilimumab at 21 days and then AF647-conjugated ipilimumab; IPIAF647: addition of AF647conjugated ipilimumab. Median fluorescence intensity (MFI) of binding assay experiment is shown on bottom table. (B) Both ipilimumab and PEEP-ipilimumab induced a decrease of fluorescence suggesting effective binding to its ligand.

We analyzed long-term memory response by challenging the mice with the tumorigenic dose of CT26 on the contralateral flank. No tumor growth was observed in mice that showed an objective response in the ipsilateral flank subjected to direct injection of native anti-CTLA4 $(n=7)$ or with injection of anti-CTLA4 PEEP $(n=8)$, while the five naive mice (with no previous treatment) showed a rapid tumor growth in all cases.

Before sacrifice, X-ray imaging showed remnant ethiodized oil uptake in the tumors injected with Pickering emulsion. In these tumors, histopathological analysis confirmed the absence of tumor cell. We observed either a fully necrotic tumor or a pseudocystic lesion. Both contained areas with optically empty, round structures measuring 30 to $60 \mu \mathrm{m}$, consistent with lipidic bodies and associated with macrophagic reaction and focal neutrophilic and lymphocytic infiltration (figure 7).

\section{DISCUSSION}

This study demonstrates that ethiodized oil and PLGA Pickering emulsions allow the loading of both murine and human anti-CTLA4 antibodies, in the internal phase of a stable water in oil radiopaque emulsion. The release of the antibodies was progressive over time, offering an opportunity to increase the local exposure to anti-CTLA4. Also, the anti-CTLA4 antibodies released by such Pickering emulsion were not altered in their structure, they kept their functional binding to CTLA4 in vitro, and their antitumor activity in vivo.

Local delivery is probably of interest when using antiCTLA4 antibodies because of their dose-efficacy-toxicity correlation thus limiting their systemic administration. ${ }^{9}$ Local delivery of therapeutic agents to a tumor usually allows for high tumorous and peritumorous concentrations while keeping the systemic exposure low. Our platform has the advantage of its versatility, with delivery being possible, percutaneously into the tumor, intra-arterially or through the lymphatic vessels. Percutaneous intratumor delivery with such drug delivery formulation will allow for longer exposure locally to the drug, but raises questions about the diffusion of the drug throughout the tumor microenvironment. Injection within the arterial tumor supply can target the entire tumor and the tumor microenvironment, which is known to be immunosuppressive.$^{30}$ Also, this ethiodized oil PLGA Pickering emulsion drug delivery platform allows for a progressive release of monoclonal antibodies over several days. This could offer an opportunity to prevent the rapid washout of ISmAbs 

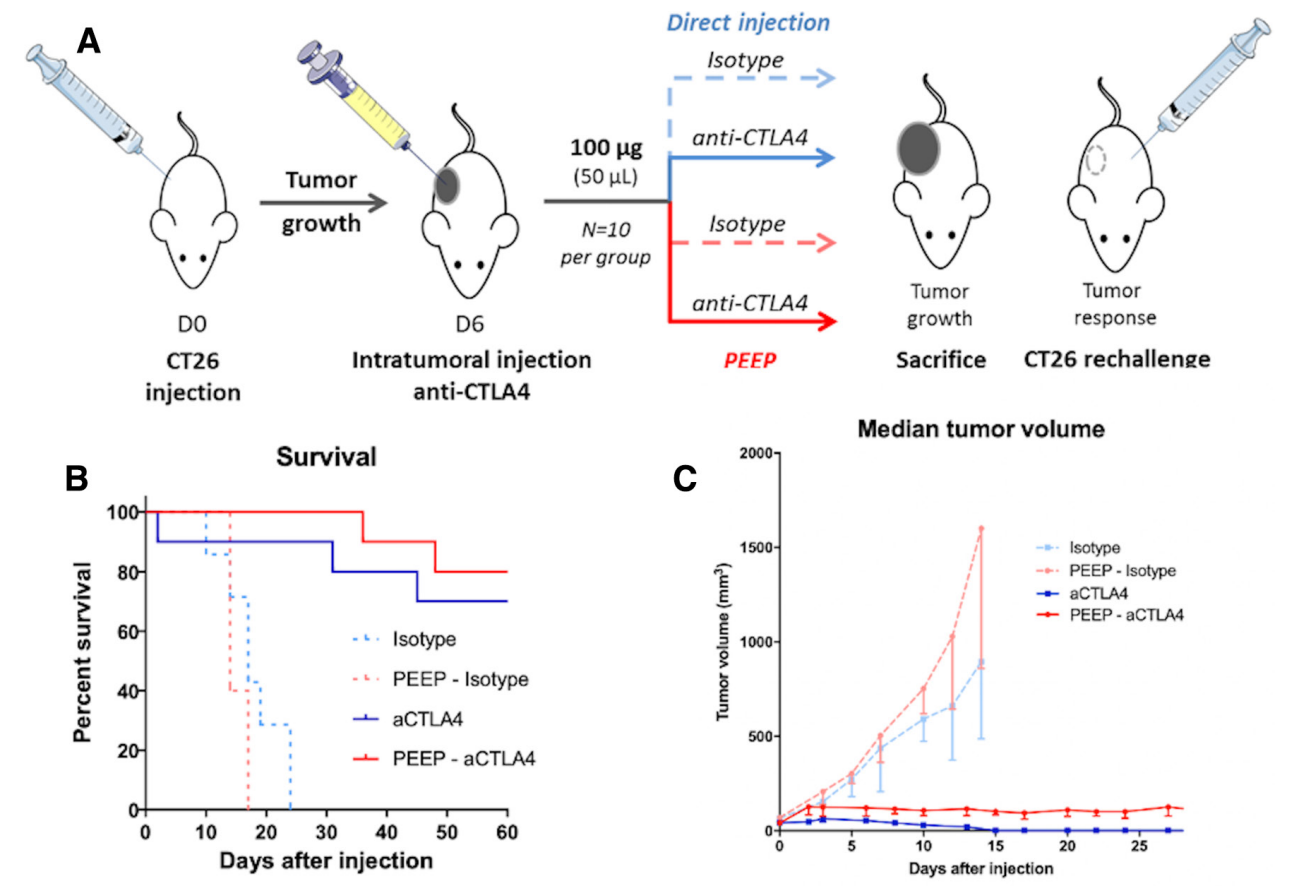

Figure 6 In vivo antitumorous activity of intratumorous anti-CTLA4 and anti-CTLA4 released from Pickering emulsions using ethiodized oil and poly-lactic-co-glycolic acid nanoparticles (PEEP). (A) In vivo experiment design with a single intratumorous injection (day 6) of anti-CTLA4 or PEEP-anti-CTLA4. (B and C) Kaplan-Meier and tumor growth curves (median and IQR of tumor sizes at different time points) according to the groups showing significant efficacy of both directly injected anti-CTLA4 and PEEP-anti-CTLA4.

from the tumor. Ethiodized oil has been demonstrated to have a temporary embolic effect related to emulsion viscosity. ${ }^{31}$ However, such intra-arterial delivery can only be done for a selected number of tumor locations (eg, hepatic, renal...), and specific studies are awaited.

We demonstrated that ethiodized oil emulsion with anti-CTLA4 is not possible without adjunct of Pickering technology and that it protects the antibody from being altered by ethiodized oil. In addition, as demonstrated previously Pickering allows for prolonged stability of the emulsion. ${ }^{27}$ A recent study has demonstrated that ipilimumab remains active for at least 4 weeks after opening of the vial, ${ }^{32}$ hence making it suitable for progressive release. Others have reported an increased therapeutic index of intratumorous immunotherapies and decreased toxicities when mixing therapeutic antibody with an oily phase too. ${ }^{13}$ In this report, the oil used was Montanide (Seppic, USA), which has also been reported to increase vaccinal effect, ${ }^{33}$ but its use might be limited as it contains surfactants. Our delivery platform is based on ethiodized oil, which is already approved for a long time in clinical practice with various delivery routes such as intra-arterial, intralymphatic and remains used for hysterosalpingography. ${ }^{34-36}$

In our in vivo experiment study, responses and survival rates offered by the intratumorous injection of the antibody alone and intratumorous injection of the Pickering emulsion were not significantly different. Beyond local efficacy on injected tumors, both anti-CTLA4 and
PEEP-anti-CTLA4 conferred an in situ vaccine protection, demonstrated by the absence of tumor regrowth in mice rechallenged with the same tumor cell line. Interestingly, the Pickering emulsion group showed an initial postinjection increase of the tumor volumes and no complete clinical tumor responses. However, the survival of these mice was equivalent to regular intratumorous anti-CTLA4 and these mice seemed to be cured. The slow degradation of the Pickering emulsion in the injected tumor was responsible for this paradoxical phenomenon. Indeed, the volume of injected ethiodized oil was still visible on X-ray imaging, and we found complete pathological responses on resection of these tumors.

We have shown that this local immunotherapy can have local efficacy against established tumors and systemic protection against tumor rechallenge. In the clinic, such efficacy could be of therapeutic interest for patients with focal relapse on anti-PD $(\mathrm{L}) 1$ monotherapy. It could also be of great interest in a neoadjuvant setting for patients bearing a localized cancer to prime the antitumor response before the surgery, to facilitate the resection of the injected lesion if some shrinkage can be obtained, and also to prevent subsequent relapses.

Radio-opacity of PEEPs is obviously of significant value when it comes to the monitoring of intratumorous and intra-arterial local injections. Indeed, this feature allows for real-time assessment during the procedure, but also after the procedure with a postoperative imaging to control what has been obtained in terms of local 

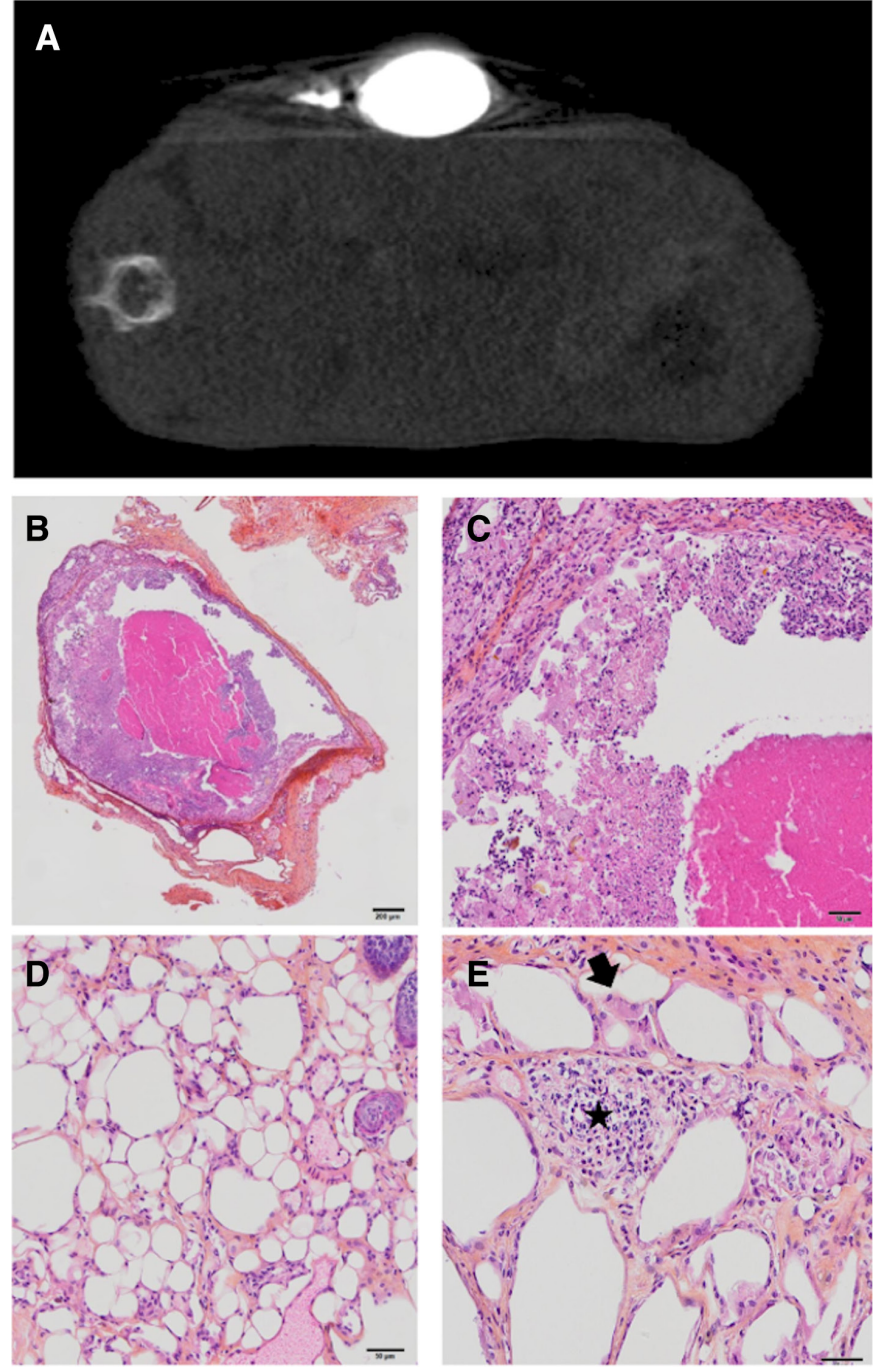

Figure 7 Radiological and pathological aspect of CT26 tumors after intratumorous injection of anti-CTLA4 Pickering emulsions using ethiodized oil and poly-lactic-co-glycolic acid nanoparticles (PEEP). (A) X-ray assessment of PEEP-anti-CTLA4 deposition in the treated tumor before mouse sacrifice, showing remnant uptake. (B, C, D and E) Histopathological assessment of CT26 tumors after injection of intratumorous injection of PEEP-anti-CTLA4 (H\&Esaffron staining). Example of a necrotic lesion surrounded by a fibrous capsule and containing a necrotic and focally inflammatory material without viable tumor cells (B scale bar: $200 \mu \mathrm{m}$ and $\mathrm{C}$ scale bar: $50 \mu \mathrm{m})$. All tumors contained areas with large, optically empty vacuoles without nuclei (D scale bar: $50 \mu \mathrm{m})$, associated with large, reactive, macrophages (E scale bar: $50 \mu \mathrm{m}$ ) (arrows), and focal neutrophilic and lymphocytic infiltration (star).

distribution of the emulsion. This radio-opacity allows for a better understanding of the drug delivery pattern and possible spatial correlation with response patterns. Indeed, recently radiopaque microsphere were released for intra-arterial therapy with the same aim, ${ }^{37}$ but today only a few chemotherapeutic agents can be loaded on these microspheres contrarily to Pickering technology that allows for delivery of various drugs.
Further research is needed to translate this work into a clinical trial, including the stability of the Pickering emulsion after passing through a catheter or on the arterial shear stress. Another limitation of our study is that no pharmacokinetic analysis was done to provide proof of longer local exposure with PEEP administration and no survival benefit over direct intratumorous injection was shown in this specific tumor model, which responds very well to anti-CTLA4 antibodies. It will be important to assess several delivery protocols, volume and frequency of injections in relationship with pharmacokinetic studies of the drug loaded in the Pickering emulsion. Also, other immunotherapies than anti-CTLA4 and other tumor models need to be evaluated to explore the potential clinical benefit of this platform for cancer immunotherapy.

In conclusion, radiopaque anti-CTLA4 ethiodized oil PLGA Pickering emulsion allows for the loading and effective release of active antibody to prime the local antitumorous immune response and generate a protective systemic immunity against the tumor. This therapeutic platform offers promising clinical applications of local immunotherapies for oncologists, radiologists, and interventional radiologists.

\section{Author affiliations}

${ }^{1}$ Interventional Radiology, Gustave Roussy, Villejuif, France

${ }^{2}$ Laboratoire de Recherche Translationnelle en Immunothérapie (LRTI), INSERM U1015, Villejuif, France

${ }^{3}$ Plateforme d'Evaluation Préclinique, AMMICA UMS 3655/US23, Gustave Roussy, Villejuif, France

${ }^{4}$ Pathology Department, Gustave Roussy, Villejuif, France

${ }^{5}$ INSERM, UMR 1015, Gustave Roussy, Villejuif, France

${ }^{6}$ Institut Galien, CNRS, Université Paris-Saclay, Châtenay-Malabry, France ${ }^{7}$ Département d'Innovation Thérapeutique et d'Essais Précoces (DITEP), Drug Development Unit, Gustave Roussy, Villejuif, France

Acknowledgements The authors acknowledge all the members of Laboratoire de Recherche Translationnelle en Immunothérapie (LRTI) and Institut Galien for facilitating this work.

Contributors All authors: conception, data analysis, writing. LT, TI, SS, LM, FD: ex vivo experiments. LT, TI, KS-LR, MP: in vivo experiments.

Funding This study is funded by the research grant of Terumo (no RE003031 GRT 2018/04).

Competing interests None declared.

Patient consent for publication Not required.

Provenance and peer review Not commissioned; externally peer reviewed.

Data availability statement Data are available on reasonable request. Data may be obtained from a third party and are not publicly available. All data relevant to the study are included in the article or uploaded as supplementary information. No clinical data were used for this publication. All preclinical data are included in the publication.

Open access This is an open access article distributed in accordance with the Creative Commons Attribution Non Commercial (CC BY-NC 4.0) license, which permits others to distribute, remix, adapt, build upon this work noncommercially, and license their derivative works on different terms, provided the original work is properly cited, appropriate credit is given, any changes made indicated, and the use is non-commercial. See http://creativecommons.org/ licenses/by-nc/4.0/.

\section{ORCID iDs}

Lambros Tselikas http://orcid.org/0000-0002-8878-4916

Aurélien Marabelle http://orcid.org/0000-0002-5816-3019 


\section{REFERENCES}

1 Hodi FS, O'Day SJ, McDermott DF, et al. Improved survival with ipilimumab in patients with metastatic melanoma. N Engl J Med 2010;363:711-23.

2 Robert C, Thomas L, Bondarenko I, et al. Ipilimumab plus dacarbazine for previously untreated metastatic melanoma. $N$ Engl J Med 2011;364:2517-26.

3 Schadendorf D, Hodi FS, Robert C, et al. Pooled analysis of long-term survival data from phase II and phase III trials of ipilimumab in unresectable or metastatic melanoma. J Clin Oncol 2015;33:1889-94.

4 Robert C, Schachter J, Long GV, et al. Pembrolizumab versus ipilimumab in advanced melanoma. N Engl J Med 2015;372:2521-32.

5 Ribas A, Puzanov I, Dummer R, et al. Pembrolizumab versus investigator-choice chemotherapy for ipilimumab-refractory melanoma (KEYNOTE-002): a randomised, controlled, phase 2 trial. Lancet Oncol 2015;16:908-18.

6 Ascierto PA, Del Vecchio M, Robert C, et al. Ipilimumab 10 mg/ $\mathrm{kg}$ versus ipilimumab $3 \mathrm{mg} / \mathrm{kg}$ in patients with unresectable or metastatic melanoma: a randomised, double-blind, multicentre, phase 3 trial. Lancet Oncol 2017;18:611-22.

7 Wang C, Hsueh AJ, Erickson GF. Lh stimulation of estrogen secretion by cultured rat granulosa cells. Mol Cell Endocrinol 1981;24:17-28.

8 Wang E, Kang D, Bae K-S, et al. Population pharmacokinetic and pharmacodynamic analysis of tremelimumab in patients with metastatic melanoma. J Clin Pharmacol 2014;54:1108-16.

9 Larkin J, Chiarion-Sileni V, Gonzalez R, et al. Five-Year survival with combined nivolumab and ipilimumab in advanced melanoma. $N$ Engl J Med 2019;381:1535-46.

10 Larkin J, Chiarion-Sileni V, Gonzalez R, et al. Combined nivolumab and ipilimumab or monotherapy in untreated melanoma. $N$ Engl $J$ Med 2015;373:23-34.

11 Marabelle A, Kohrt H, Caux C, et al. Intratumoral immunization: a new paradigm for cancer therapy. Clin Cancer Res 2014;20:1747-56.

12 Marabelle A, Andtbacka R, Harrington K, et al. Starting the fight in the tumor: expert recommendations for the development of human intratumoral immunotherapy (HIT-IT). Ann Oncol 2018;29:2163-74.

13 Fransen MF, van der Sluis TC, Ossendorp F, et al. Controlled local delivery of CTLA-4 blocking antibody induces CD8+ T-celldependent tumor eradication and decreases risk of toxic side effects. Clin Cancer Res 2013;19:5381-9.

14 Marabelle A, Kohrt H, Sagiv-Barfi I, et al. Depleting tumor-specific Tregs at a single site eradicates disseminated tumors. J Clin Invest 2013;123:2447-63.

15 Aznar MA, Tinari N, Rullán AJ, et al. Intratumoral delivery of Immunotherapy-Act locally, think globally. J Immunol 2017;198:31-9.

16 van Hooren L, Sandin LC, Moskalev I, et al. Local checkpoint inhibition of CTLA-4 as a monotherapy or in combination with antiPD1 prevents the growth of murine bladder cancer. Eur J Immunol 2017;47:385-93.

17 Sandin LC, Eriksson F, Ellmark P, et al. Local CTLA4 blockade effectively restrains experimental pancreatic adenocarcinoma growth in vivo. Oncoimmunology 2014;3:e27614.

18 Marabelle A, Kohrt H, Levy R. Intratumoral anti-CTLA-4 therapy: enhancing efficacy while avoiding toxicity. Clin Cancer Res 2013;19:5261-3.
$19 \mathrm{Xu} \mathrm{W}$, Atkinson VG, Menzies AM. Intratumoural immunotherapies in oncology. Eur J Cancer 2020;127:1-11.

20 Ray A, Williams MA, Meek SM, et al. A phase I study of intratumoral ipilimumab and interleukin-2 in patients with advanced melanoma. Oncotarget 2016;7:64390-9.

21 de Baere T, Arai Y, Lencioni R, et al. Treatment of liver tumors with lipiodol TACE: technical recommendations from experts opinion. Cardiovasc Intervent Radiol 2016;39:334-43.

22 Raoul J-L, Forner A, Bolondi L, et al. Updated use of TACE for hepatocellular carcinoma treatment: how and when to use it based on clinical evidence. Cancer Treat Rev 2019;72:28-36.

23 Roche A, Girish BV, de Baère T, et al. Trans-catheter arterial chemoembolization as first-line treatment for hepatic metastases from endocrine tumors. Eur Radiol 2003;13:136-40.

24 Benitez JC, Mezquita L, Colle R, et al. Hepatic intra-arterial chemotherapy with immunotherapy in NSCLC. J Thorac Oncol 2019;14:e215-6.

25 Deschamps F, Harris KR, Moine L, et al. Pickering-Emulsion for liver Trans-Arterial Chemo-Embolization with oxaliplatin. Cardiovasc Intervent Radiol 2018;41:781-8.

26 Deschamps F, Moine L, Isoardo T, et al. Parameters for stable waterin-oil lipiodol emulsion for liver Trans-Arterial Chemo-Eembolization. Cardiovasc Intervent Radiol 2017;40:1927-32.

27 Deschamps F, Isoardo T, Denis S, et al. Biodegradable Pickering emulsions of lipiodol for liver trans-arterial chemo-embolization. Acta Biomater 2019;87:177-86.

28 Le-Minh V, Tran NT, Makky A, et al. Capillary zone electrophoresisnative mass spectrometry for the quality control of intact therapeutic monoclonal antibodies. J Chromatogr A 2019;1601:375-84.

29 Selby MJ, Engelhardt JJ, Quigley M, et al. Anti-Ctla-4 antibodies of IgG2a isotype enhance antitumor activity through reduction of intratumoral regulatory T cells. Cancer Immunol Res 2013;1:32-42.

30 Pitt JM, Marabelle A, Eggermont A, et al. Targeting the tumor microenvironment: removing obstruction to anticancer immune responses and immunotherapy. Ann Oncol 2016;27:1482-92.

31 de Baere T, Dufaux J, Roche A, et al. Circulatory alterations induced by intra-arterial injection of iodized oil and emulsions of iodized oil and doxorubicin: experimental study. Radiology 1995;194:165-70.

32 Bardo-Brouard P, Vieillard V, Shekarian T, et al. Stability of ipilimumab in its original vial after opening allows its use for at least 4 weeks and facilitates pooling of residues. Eur J Cancer 2016;58:8-16.

33 Aucouturier J, Dupuis L, Deville S, et al. Montanide Isa 720 and 51: a new generation of water in oil emulsions as adjuvants for human vaccines. Expert Rev Vaccines 2002;1:111-8.

34 Pieper CC, Hur S, Sommer C-M, et al. Back to the future: lipiodol in Lymphography-From diagnostics to theranostics. Invest Radiol 2019;54:600-15.

$35 \mathrm{Yu}$ SCH, Chan SL, Lee KF, et al. Ablative chemoembolization for hepatocellular carcinoma: a prospective phase I case-control comparison with conventional chemoembolization. Radiology 2018;287:340-8.

36 Eisen D, Goldstein J. Lipiodol intravasation during uterosalpingography with pulmonary complications. Radiology 1945;45:603-7.

37 Reicher J, Mafeld S, Priona G, et al. Early experience of Trans-arterial chemo-embolisation for hepatocellular carcinoma with a novel radiopaque bead. Cardiovasc Intervent Radiol 2019;42:1563-70. 